\title{
HAS A STANDARD MODEL SOLUTION TO THE SOLAR NEUTRINO PROBLEM BEEN FOUND?
}

\author{
JOHN N. BAHCALL ${ }^{*} \dagger$, C. A. BARNES ${ }^{\ddagger}$, J. CHRISTENSEN-DALSGAARD ${ }^{\S}$, \\ B. T. CLEVELAND , S. DEGL'INNOCENTI",2, B. W. FILIPPONE ${ }^{3}$, \\ A. GLASNER ${ }^{*}, 2$, R. W. KAVANAGH ${ }^{3}$, S. E. KOONIN ${ }^{3}$, K. LANDE ${ }^{5,2}$, \\ K. LANGANKE ${ }^{3}$, P. D. PARKER ${ }^{\dagger} \dagger, 2$ M. H. PINSONNEAULT $\ddagger^{\ddagger}, 2$, \\ C. R. PROFFITT ${ }^{10,2}$, and T. SHOPPA ${ }^{3}$
}

\begin{abstract}
The claim by Dar and Shaviv that they have found a standard model solution to the solar neutrino problem is based upon an incorrect assumption made in extrapolating nuclear cross sections and the selective use of a small fraction of the nuclear physics and of the neutrino data. In addition, five different solar model codes show that the rate obtained for the chlorine experiment using the Dar-Shaviv stated parameters differs by at least $14 \sigma$ from the observed rate.
\end{abstract}

\section{Introduction}

In a widely circulated preprint, Dar and Shavivt claim to have provided a standard solar model solution to the solar neutrino problem. Their preprint has aroused sufficient curiosity that we feel compelled to point out its most obvious defects, although we do not plan to publish our response in a refereed journal unless the Dar and Shaviv preprint is also published in a refereed journal.

To summarize our response, Dar and Shaviv have not solved the solar neutrino problem. They have made an incorrect assumption in extrapolating nuclear cross sections and have used nuclear and neutrino data selectively. Most surprisingly, their solar model results are not reproducible. We obtain from five independent solar neutrino codes and the Dar-Shaviv input parameters, neutrino fluxes that are inconsistent with the values reported by Dar and Shaviv. With the Dar

*Institute for Advanced Study, School of Natural Sciences, Princeton, NJ 08540

$\dagger$ Also, Institute for Nuclear Theory, University of Washington, Seattle, WA 98195

$\ddagger$ W. K. Kellogg Radiation Laboratory, Caltech, Pasadena, CA 91125

$\S$ Institut for Fysik og Astronomi, Aarhus Universitet, DK-8000, Aarhus C, Denmark

ฯ Physics Department, University of Pennsylvania, Philadelphia, PA 19104-6394

"Departimento di Fisica dell'Universita di Ferrara and Instituto Nazionale di Fisica Nucleare, Sezione di Ferrara, I-44100, Ferrara, Italy

** Racah Institute of Physics, Hebrew University, Jerusalem, Israel and Department of Astronomy and Astrophysics, University of Chicago, Chicago, IL 60637

${ }^{\dagger \dagger}$ Physics Department, Yale University, New Haven, CT 06511

$¥$ Department of Astronomy, Ohio State University, Columbus, OH 43210

Computer Sciences Corporation, IUE Observatory, Code 684.9, Goddard Space Flight Center, Greenbelt, $M D 20771$ 
and Shaviv input parameters, all four solar neutrino experiments differ from the solar model results (obtained with the five different codes) by more than $14 \sigma$ for the chlorine experiment $[($ Rate (theory) - Rate $(\exp )) / \sigma(\exp )]$, , m more than $4 \sigma$ for the Kamiokande experiment, 3 more than $4 \sigma$ for the GALLEX (gallium) experiment, 1 and more than $3 \sigma$ for the SAGE (gallium) experiment 5

This response is organized as follows. In $\S 1$, we comment on the fact that Dar and Shaviv chose to compare their results with only a small part of the available chlorine solar-neutrino data. In $\S 2$, we point out that they made an ad hoc and incorrect assumption in extrapolating the nuclear physics data and compared their results with only a small fraction of the available experiments. In $\S 3$, we show that their solar model calculations are in disagreement with calculations made using five other well-tested codes.

\section{Choosing Part of the Chlorine Solar-Neutrino Experimental Data}

Dar and Shaviv chose to consider (see their Figure 1) only four years of data from the chlorine solar neutrino experiment beginning in 1987, although 23 years of data have been reported ${ }^{2}$. For the period beginning in 1987, the experimental measurement of the chlorine rate is $2.8 \pm 0.3 \mathrm{SNU}$. The measured rate for the entire period for which data has been reported is $2.28 \pm 0.23 \mathrm{SNU}$. The measured rate in the chlorine experiment, during the short period considered by Dar and Shaviv, is still more than $4 \sigma$ less than their calculated result of $4.2 \mathrm{SNU}$ and is more than $14 \sigma$ from the value we calculate (see $\S 3$ and Table 1 below) for their stated parameters.

\section{Extrapolating Nuclear Cross Sections with an ad hoc As- sumption and Using Only Part of the Experimental Data}

Dar and Shaviv claim that by replacing the conventionalo representation of the extra energy dependence (in addition to the point-nuclei barrier penetration factor) of the nuclear cross section factor by another smoothly-varying, parameterized function, they obtain a very different extrapolation to low energies for the rate of the ${ }^{3} \mathrm{He}(\alpha, \gamma)^{7} \mathrm{Be}$ reaction. Of course, both procedures must yield the same answer if they are both applied in an unbiased way to the same experimental data set and all the energy dependences are included (see below). For the reactions discussed by Dar and Shaviv, the existing data sets determine well the extrapolations.

Dar and Shaviv, however, assumed incorrectly that the only energy-dependent effect besides point-nuclei barrier penetration is nuclear size, and then compared their results with only two of the nine published experiments. Their selective use of data and this incorrect assumption explain why the Dar and Shaviv answer for $S(0)$ differs from the standard value obtained by nuclear physicists. We explain in more detail below.

For extrapolation of nuclear cross section data to stellar energies, it is con- 
ventional to define the astrophysical S-factor,

$$
S(E)=\sigma(E) E \exp \{2 \pi \eta(E)\} .
$$

The Sommerfeld parameter is given by

$$
\eta(E)=\frac{Z_{1} Z_{2} e^{2}}{\hbar v}
$$

where $v$ is the relative velocity of the two particles in the entrance channel, $E$ is their relative energy, and $Z_{1}, Z_{2}$ are their charges. The form of Eq. (1) accounts explicitly for the energy dependence of s-wave tunneling through the Coulomb barrier of two pointlike particles and a kinematic flux factor. In the absence of near-threshold resonances, the energy dependence of the S-factor is expected to be small at low energies. However, in order to carry out a reliable extrapolation, the energy-dependent effects that are not accounted for in Eq. (1) must be allowed to show up in $S(E)$. These effects include nuclear structure, the strong interaction, energy dependent operators in the transition matrix elements, antisymmetrization between the colliding nucleons, finite nuclear size, the finalstate phase space, and the contributions from other partial waves. In fact, for all the reactions of importance for the solar $p-p$ chain, the observed energy dependence of the various reactions agrees well with that calculated in theoretical models which account explicitly for the known nuclear effects that are omitted from Eq. (1). In particular, it was demonstrated more than thirty years ago that the complete Coulomb wave function provides a good description of the measured cross section factor for the ${ }^{2} H(p, \gamma)^{3} H e$ reaction down almost to solar thermal energies $(16 \mathrm{keV})$.

Dar and Shaviv have chosen to factor out a slightly different energy dependence from the cross section data by defining a modified S-factor, $\bar{S}(E)$, as

$$
\bar{S}(E)=\sigma(E) E \exp \{2 \pi \eta(E)[1+2 / \pi(2 \sqrt{x}-\arcsin \sqrt{x}-\sqrt{x(1-x)}))\},
$$

with $x=E / E_{c}$ and the Coulomb energy $E_{c}=Z_{1} Z_{2} e^{2} / R$ at a radius parameter $R$. With this definition, $\bar{S}(E)$ attempts to account for the finite size of the nuclei. However, $\bar{S}(E)$ is still expected to be dependent on $E$, because of the other effects listed above that introduce an energy dependence in addition to the finite size of the nucleus. The theoretical models that have been used previously 10 to extrapolate the cross section data to solar energies properly take account of the finite nuclear size effects along with the other effects discussed above. Obviously both definitions, Eqs. (1) and (3), must lead to the same results for the low energy S-factor when proper account is taken of the additional energy dependence not included explicitly in the respective equations.

Dar and Shaviv did not take account of the additional energy dependences nor of all of the available nuclear physics data. For the determination of the lowenergy cross section factor, $\mathrm{S}_{34}$, for the ${ }^{3} \mathrm{He}(\alpha, \gamma)^{7} \mathrm{Be}$ reaction, Dar and Shaviv apparently adjusted the radius parameter $R$ (see Eq. 3 above) so that the energy 
dependence of $\bar{S}(E)$ is mostly removed for two of the nine 10 existing experiments. (They seem not to have noticed that the value of $R$ that they obtain is very different from the measured radius of $2.8 \mathrm{fm}$ determined by electron scattering.) They did not allow for the other energy dependences discussed above and they only took account of two of the experiments.

For the determination of the cross-section factor for the ${ }^{7} B e(p, \gamma)^{8} B$ reaction, $\mathrm{S}_{17}$, Dar and Shaviv first remove the contribution from d-wave capture (based on previous calculations of this single component of the energy dependence). However, the $\mathrm{d}$-wave capture contributes about $6 \%$ to the S-factor at the most effective energy for the reaction (about $20 \mathrm{keV}$ ) and must be added to the computed s-capture rate. In addition, previous calculations 11 demonstrate that there are significant further contributions to the energy dependence for $\mathrm{S}_{17}$ that must be included in order to make a reliable extrapolation. When these additional effects are included in the extrapolation of $\bar{S}_{17}(E)$ (Eq. 3 above), the results must agree with the standard extrapolations.

Dar and Shaviv cited the preliminary Coulomb dissociation work described in preprint form 12 as evidence for a lower-than-standard value for the crucial cross section factor for the ${ }^{7} B e(p, \gamma){ }^{8} B$ reaction. When the $E 2$ contribution to this reaction is taken into account, 13 the preliminary Coulomb-dissociation value differs from the six direct measurements 10 of the ${ }^{7} B e(p, \gamma)^{8} B$ cross section by a factor of two while the estimated uncertainty 8 in direct measurements is only $11 \%$. Moreover, there are still some unanswered questions about the application of the Coulomb-dissociation method for determining radiative capture cross sections, aside from the experimental difficulties inherent in covering a sufficient range in energy and angle to validate the reliability of any inferences.

For other nuclear reactions, Dar and Shaviv have used the low-energy cross section factors from an earlier review 14 which provided fitting formulae suitable for use at temperatures $\left(\sim 10^{9} \mathrm{~K}\right)$ much higher than are reached in the sun $\left(\sim 10^{7} \mathrm{~K}\right)$. The quantitative effect of these approximations is difficult to estimate, especially since other authors (see section 4) use-for solar calculations-explicit formulae that are suitable for the lower solar temperatures. However, an approximate discussion of using the fitting formulae for higher temperatures at solar temperatures has been given 15 ; the principal effects of the high-temperature formulae are in the direction to decrease the predicted ${ }^{7} \mathrm{Be}$ and ${ }^{8} \mathrm{~B}$ neutrino fluxes.

Dar and Shaviv discuss at some length the fact that the Debye-Hückel approximation to the screened nuclear potential is not correct everywhere in the sun. It is not clear what they recommend (although they say the effect is small), nor if they are aware that modern screening calculations go well beyond what they discuss. 16.17

\section{Solar Model Calculations}

Six authors of this paper (Bahcall, Christensen-Dalsgaard, Degl'Innocenti, Glasner, Pinsonneault, and Proffitt) have repeated the solar model calculations of Dar and Shaviv using the non-standard parameters that Dar and Shaviv chose, namely, a solar luminosity of $3.826 \times 10^{33} \mathrm{erg} \mathrm{s}^{-1}$ and low energy cross-section 
factors of $S_{34}(0)=0.45$ kev-b and $S_{17}(0)=17 \mathrm{eV}-\mathrm{b}$. Dar and Shaviv did not specify in their preprint many of the important input quantities in their model; they did not state what they used for the element abundances, the radiative opacities, the equation of state, and the neutrino cross sections. They did not say which of the several available perscriptions for diffusion they used. We have therefore carried out calculations using a variety of different choices for these quantities, namely, the choices made previously as their best estimates by the six different authors who used five independent stellar evolution codes. 1822

The results are shown in Table 1 . The first column identifies the computer code used in constructing the solar model; the second column indicates whether or not particle diffusion was included. The third, fourth and fifth columns give the calculated rates predicted for the chlorine, gallium, and Kamiokande solar neutrino experiments, respectively. The numbers in parentheses indicate the number of standard deviations quoted by the experimentalists (adding statistical and systematic errors quadratically) by which the calculated rates differ from the measured rates. For gallium, we have compared with the most recent GALLEX determination (which has smaller quoted uncertainties). The last two columns of Table 1 give the fluxes of ${ }^{13} \mathrm{~N}$ and ${ }^{15} \mathrm{O}$ solar neutrinos. Some details regarding the solar models are given in the footnotes to the table.

The calculations reported by Dar and Shaviv, the first row of Table 1, predict a rate that differs by $8 \sigma$ from the average chlorine experimental value. Even if their calculations and assumptions were correct (and we think they are not), then they have not solved the solar neutrino problem unless one completely disregards the chlorine solar neutrino experiment.

In rows two through four we give results obtained with five different computer codes that have been intercompared in the literature with other standard models; all of these codes have previously been shown to give the same results for the same input parameters and to give results that agree well with still other welltested solar model codes. For the Dar and Shaviv parameters, all of the five well-calibrated solar codes give results very close to each other, as they must, but they all differ significantly from the Dar and Shaviv results. The relatively small differences between the five well-calibrated solar models are primarily due to the treatment of particle diffusion (see column two). This is a significant physical effect that is somewhat difficult to include in solar model calculations, but which has been taken into account in the most recent and advanced solar model computer programs.

For the Dar and Shaviv parameters, the five well-calibrated models give for

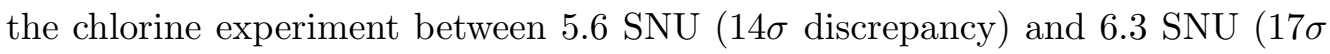
discrepancy) depending upon whether or not particle diffusion is included. This contrasts with the value of $4.2 \mathrm{SNU}$ reported by Dar and Shaviv. All five of the models predict about $125 \mathrm{SNU}$ ( $4 \sigma$ discrepancy) for gallium, which contrasts with the Dar and Shaviv value of 109 SNU. Finally, the ${ }^{8} \mathrm{~B}$ flux in the five well-calibrated solar models is between $4 \sigma$ and $6 \sigma$ from the Kamiokande result (depending upon whether or not particle diffusion is included), while the Dar and Shaviv result agrees almost exactly with the Kamiokande measurement. 
Even more striking differences exist for the ${ }^{13} \mathrm{~N}$ and ${ }^{15} \mathrm{O}$ neutrino fluxes, for which the Dar and Shaviv values differ by an order of magnitude from the values obtained in the other programs. Their flux for the ${ }^{13} \mathrm{~N}$ neutrinos is low by about a factor of 6 and their ${ }^{15} \mathrm{O}$ flux is low by about a factor of 15 .

None of the well-tested solar codes that we have used are able to reproduce the Dar and Shaviv results for neutrino fluxes.

\section{Summary}

Dar and Shaviv did not succeed in solving the solar neutrino problem despite the introduction of an ad hoc assumption, the selective treatment of data, and the incorrect extrapolation of some nuclear cross sections. Their failure to solve the problem is not surprising since it has been demonstrated elsewhere 23 that the chlorine and the Kamiokande experiments are inconsistent with one another if one assumes (on the basis of standard electroweak theory) that the shape of the ${ }^{8} \mathrm{~B}$ neutrino spectrum is the same in the laboratory and in the sun.

Several of the authors (JNB, SD, AG, KL, PDP, MHP, and CRP) thank the Institute for Nuclear Theory at the University of Washington for its hospitality and the Department of Energy for partial support during the completion of this work.

\section{References}

1. A. Dar, and G. Shaviv, Phys. Rev. Lett., preprint submitted (1994).

2. R. Davis Jr., in Frontiers of Neutrino Astrophysics, ed. Y. Suzuki, and K.

Nakamura (Tokyo: Universal Academy Press, Inc., 1993), p. 47.

3. K. S. Hirata, Phys. Rev. D 44, 2241 (1991); Y. Suzuki, in Frontiers of Neutrino Astrophysics, ed. Y. Suzuki, and K. Nakamura (Tokyo: Universal Academy Press, Inc., 1993), p. 47.

4. P. Anselmann, Phys. Lett. B 314, 445 (1993); P. Anselmann, et al., Phys. Lett., submitted, February (1994).

5. A. I. Abazov, Nucl. Phys. B (Proc. Supppl.) 19, 84 (1991).

6. W. A. Fowler, Rev. Mod. Phys. 56, 149 (1984).

7. G. M. Griffiths, M. Lal, and C. D. Scarfe, Can. J. Phys. 41, 724 (1963).

8. C. W. Johnson, E. Kolbe, S. E. Koonin, and K. Langanke, Astrophys. J. 392, 320 (1992).

9. T. Kajino and A. Arima, Phys. Rev. Lett. 52, 739 (1984).

10. P. D. Parker, and C. Rolfs, C. in The Solar Interior and Atmosphere, ed. A.

Cox, W. C. Livingston, and M. S. Matthews (Tucson: University of Arizona, 1991), p. 31

11. R. D. Williams, and S. E. Koonin, Phys. Rev. C 23, 2773 (1981).

12. T. Motobayashi, et al., Phys. Rev. Lett., submitted (1994).

13. K. Langanke, and T. Shoppa (CalTech preprint), Phys. Rev. C, in press (April, 1994).

14. G. R. Caughlan, and W. A. Fowler, At. Data Nucl. Data Tables 40, 283 (1988).

15. J. N. Bahcall, and M. H. Pinsonneault, Rev. Mod. Phys. 64, 885 (1992).

16. H. E. DeWitt, H. C. Graboske, and M. S. Cooper, Astrophys. J. 181, 439 (1973). 
17. C. A. Carraro, A. Schafer, and S. E. Koonin, Astrophys. J. 331, 565 (1988).

18. J. N. Bahcall, and M. H. Pinsonneault, in preparation (1994).

19. C. R. Proffitt, Astrophys. J. 425, 849 (1994).

20. V. Castellani, S. Degl'Innocenti, and G. Fiorentini, Astron. and Astrophys. 271, 601 (1993).

21. J. Christensen-Dalsgaard, C. R. Proffitt, and M. J. Thompson, Astrophys. J. 403, L75 (1993).

22. J. N. Bahcall, and A. Glasner, Astrophys. J., submitted (1994).

23. J. N. Bahcall, and H. A. Bethe, Phys. Rev. D 47, 1298 (1993). 
Table 1

Solar Model Results for Dar and Shaviv Parameters

\begin{tabular}{lcccccc}
\hline \hline \multicolumn{1}{c}{ Solar Code } & Diffusion & $\begin{array}{c}{ }^{37} \mathrm{Cl} \\
(\mathrm{SNU})\end{array}$ & $\begin{array}{c}{ }^{71} \mathrm{Ga} \\
(\mathrm{SNU})\end{array}$ & ${ }^{8} \mathrm{~B}^{\dagger}$ & ${ }^{13} \mathrm{~N}^{*}$ & ${ }^{2} \mathrm{O}^{*}$ \\
\hline Dar and Shaviv & Yes? & $4.2(8 \sigma)$ & $109(2.6 \sigma)$ & $2.8(0 \sigma)$ & 0.7 & 0.2 \\
YALE $^{\mathrm{a}}$ & Yes & $6.3(17 \sigma)$ & $125(4 \sigma)$ & $4.2(6 \sigma)$ & 5.9 & 5.1 \\
AARHUS $^{\mathrm{b}}$ & No & $5.6(14 \sigma)$ & $122(4 \sigma)$ & $3.6(4 \sigma)$ & 5.4 & 5.4 \\
& Yes & $6.1(16 \sigma)$ & $124(4 \sigma)$ & $4.0(5 \sigma)$ & 5.8 & 5.8 \\
Proffitt $^{\mathrm{c}}$ & Yes & $6.3(17 \sigma)$ & $126(4 \sigma)$ & $4.2(6 \sigma)$ & 6.3 & 5.5 \\
FRANEC $^{\mathrm{d}}$ & No & $5.8(15 \sigma)$ & $123(4 \sigma)$ & $3.9(5 \sigma)$ & 4.9 & 4.2 \\
ASTRA $^{\mathrm{e}}$ & No & $5.7(15 \sigma)$ & $123(4 \sigma)$ & $3.7(4 \sigma)$ & 4.2 & 3.5 \\
\hline
\end{tabular}

$\dagger_{\text {Units: }} 10^{6} \mathrm{~cm}^{-2} \mathrm{~s}^{-1} ;{ }^{*}$ Units: $10^{8} \mathrm{~cm}^{-2} \mathrm{~s}^{-1}$.

a cf. Ref. 18 Includes helium and heavy element diffusion, as well as other improvements.

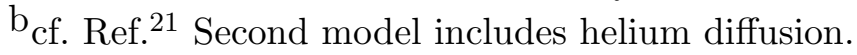

${ }^{c}$ cf. Ref 19 Includes helium and heavy element diffusion.

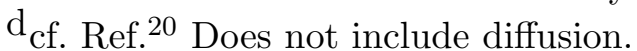

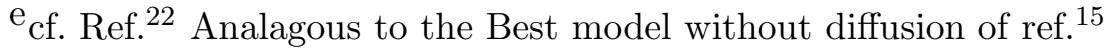

